\title{
Lower Bound Estimate of Blow Up Time for the Porous Medium Equations under Dirichlet and Neumann Boundary Conditions
}

\author{
XUE Yingzhen*
}

School of Business, Xi'an International University, Xi'an 710077, China

Received 1 August 2020; Accepted 31 October 2020

\begin{abstract}
In this paper, we establish the lower bounds estimate of the blow up time for solutions to the nonlocal cross-coupled porous medium equations with nonlocal source terms under Dirichlet and Neumann boundary conditions. The results are obtained by using some differential inequality technique.
\end{abstract}

AMS Subject Classifications: 35K20, 35K55, 35K60, 35K65

Chinese Library Classifications: O175.26

Key Words: Lower bounds; Blow up time; Nonlocal source terms; Dirichlet and Neumann boundary conditions.

\section{Introduction}

In this article, we consider the lower bound of blow up time for solutions of the nonlocal cross-coupled porous medium equations

$$
\begin{aligned}
& u_{t}=\Delta u^{m}+\int v^{p} \mathrm{~d} x, \quad(x, t) \in \Omega \times\left(0, t^{\star}\right), \\
& v_{t}=\Delta v^{n}+\int u^{q} \mathrm{~d} x, \quad(x, t) \in \Omega \times\left(0, t^{\star}\right),
\end{aligned}
$$

and continuous bounded initial values

$$
u(x, 0)=u_{0}(x), \quad v(x, 0)=v_{0}(x), \quad x \in \Omega,
$$

under Dirichlet boundary condition

$$
u(x, t)=v(x, t)=0, \quad(x, t) \in \partial \Omega \times\left(0, t^{\star}\right),
$$

*Corresponding author. Email addresses: xueyingzhen@126.com, xueyingzhen@xaiu.edu.cn (Y. Xue) 
or Neumann boundary condition

$$
\frac{\partial u^{m}}{\partial v}=l u, \quad \frac{\partial v^{n}}{\partial v}=l v, \quad(x, t) \in \partial \Omega \times\left(0, t^{\star}\right),
$$

where $\Omega \in R^{3}$ is a bounded region of $\partial \Omega$ with a smooth boundary, and satisfies that $p>m>1, q>n>1, v$ is the unit external normal vector in the external normal direction of $\partial \Omega$. There are many research achievements on the lower bound estimation of blow up time for the solution of a single porous media equation, see, e.g., [1-3]. Liu, et al. [1] studied the following nonlocal porous equation with Dirichlet boundary conditions

$$
u_{t}=\Delta u^{m}+u^{p} \int u^{q} \mathrm{~d} x, \quad(x, t) \in \Omega \times\left(0, t^{\star}\right) .
$$

They have obtained the lower bound of the blow up time of the solution which

$$
t^{\star} \geq C_{7}\left[\int_{\Omega} u_{0}^{\alpha}(p+q-1) \mathrm{d} x\right]^{-C_{6}},
$$

and homogeneous Neumann boundary conditions, the lower bound of the blow up time of the solution which

$$
t^{\star} \geq \int_{\eta(0)}^{\infty} \frac{\mathrm{d} \xi}{K_{5} \xi^{\frac{\alpha+1}{\alpha}}+K_{6} \xi^{\frac{(\alpha+1)(p+q-1)}{\alpha(p+q-1)-(p+q-m)}}} .
$$

Liu [2] considered Eq. (1.6) with Robin boundary conditions, they have obtained the lower bound of the blow up time of the solution which

$$
t^{\star} \geq \int_{\phi(0)}^{\infty} \frac{\mathrm{d} \eta}{m s|\Omega| K_{4} \eta^{\frac{m s+s}{m s+m-1}}+m s|\Omega| K_{5} \eta^{\frac{m s+s}{n(m-1)(s+1)}}} .
$$

Fang and Chai [3] studied Eq. (1.6) with Neumann boundary conditions

$$
\frac{\partial u^{m}}{\partial v}=l u, \quad(x, t) \in \partial \Omega \times\left(0, t^{\star}\right),
$$

the lower bound of the blow up time of the solution which

$$
t^{\star} \geq \int_{\eta(0)}^{\eta(t)} \frac{\mathrm{d} \xi}{C_{1} \xi+C_{2} \xi^{\frac{\alpha(p+q-1)-(m-1)}{\alpha(p+q-1)}}+C_{2} \xi^{\frac{(\alpha+1)}{\alpha}}+C_{3} \xi^{\frac{(\alpha+1)(p+q-1)}{\alpha(p+q-1)-(p+q-m)}}},
$$

when $l>0$ of (1.5). The lower bound of the blow up time of the solution which

$$
t^{\star} \geq \int_{\eta(0)}^{\eta(t)} \frac{\mathrm{d} \xi}{K_{1} \xi^{\frac{(\alpha+1)}{\alpha}}+K_{2} \xi^{\frac{(\alpha+1)(p+q-1)}{\alpha(p+q-1)-(p+q-m)}}},
$$

when $l<0$ of Eq. (1.5). 
To sum up, most of the existing results focus on a single equation,However, studies on the lower bound of blow up time for the equation set (1.1)-(1.5) have not been found. Blow-up at a finite time and lower bound of blowing up time of solution for the parabolic equations are studied in $[4,5]$. The lower bound of blowing up time for solutions of other similar equations or equation set is shown in [6-9].

Inspired by [4-5], this paper studies the lower bound estimation of blowing up time for the solutions of the porous media equation set (1.1)-(1.5) with non-local source crosscoupling and $m>1, n>1$ with relevant formulas and some basic inequalities in [9].

\section{The some inequalities}

This part introduces some important inequalities used in this paper.

Lemma 2.1. (Membrane inequality)

$$
\lambda \int_{\Omega} \omega^{2} \mathrm{~d} x \leq \int_{\Omega}|\nabla \omega|^{2} \mathrm{~d} x
$$

where $\lambda$ is the first eigenvalue of $\Delta \omega+\lambda \omega=0, \omega>0, x \in \Omega$, and $\omega=0, x \in \partial \Omega$.

Lemma 2.2. ([9]) Let $\Omega$ be the bounded star region in $R^{N}$, and $N \geq 2$. Then

$$
\int_{\partial \Omega} u^{n} \mathrm{~d} \xi \leq \frac{N}{\rho_{0}} \int_{\Omega} u^{n} \mathrm{~d} x+\frac{n d}{\rho_{0}} \int_{\Omega} u^{n-1}|\nabla u| \mathrm{d} x .
$$

Lemma 2.3. (Special Young inequality) Let $\gamma$ be an arbitrary constant, and $0<x<1$. Then

$$
a^{x} b^{y}=(\gamma a)^{x}\left(\frac{b^{\frac{y}{1-x}}}{\gamma^{\frac{x}{1-x}}}\right)^{1-x} \leq \gamma x a+(1-x) \gamma^{\frac{x}{x-1}} b^{\frac{y}{1-x}}, \quad(a, b>0) .
$$

\section{Lower bound of blow up time under Dirichlet boundary con- ditions}

The lower bound of blow up time for solutions of equations under Dirichlet boundary conditions is discussed below.

Theorem 3.1. Defines auxiliary functions

$$
J(t)=\int_{\Omega}\left(u^{s}+v^{s}\right) \mathrm{d} x,
$$

for $s>\max \{1, p, q, m-1, n-1,3 p-2,3 q-2\}$. If $(u, v)$ is a non-negative classical solution of $e$ quation set (1.1)-(1.5) and blow up occurs in the sense of measure $J(t)$ at time $t^{\star}$, then the lower bound of $t^{\star}$ is

$$
\int_{J(0)}^{\infty} \frac{\mathrm{d} \eta}{K_{1} \eta^{\beta_{1}}+K_{2} \eta}
$$


where $J(0)=\int_{\Omega}\left(u_{0}^{s}+v_{0}^{s}\right) \mathrm{d} x$, The normal number $K_{1}, K_{2}, \beta_{1}$ is given in the following proof.

Proof. Note that

$$
\begin{aligned}
& J^{\prime}(t)= s \int_{\Omega} u^{s-1} u_{t} \mathrm{~d} x+s \int_{\Omega} v^{s-1} v_{t} \mathrm{~d} x \\
&= s \int_{\Omega} u^{s-1} \Delta u^{m} \mathrm{~d} x+s \int_{\Omega} u^{s-1} \int_{\Omega} v^{p} \mathrm{~d} x \mathrm{~d} x \\
& \quad+s \int_{\Omega} v^{s-1} \Delta v^{n} \mathrm{~d} x+s \int_{\Omega} v^{s-1} \int_{\Omega} u^{q} \mathrm{~d} x \mathrm{~d} x \\
&=-m s(s-1) \int_{\Omega} u^{s+m-3}|\nabla u|^{2} \mathrm{~d} x+s \int_{\partial \Omega} u^{s-1} \frac{\partial u^{m}}{\partial v} \mathrm{~d} \xi+s \int_{\Omega} u^{s-1} \mathrm{~d} x \int_{\Omega} v^{p} \mathrm{~d} x \\
& \quad-n s(s-1) \int_{\Omega} v^{s+n-3}|\nabla v|^{2} \mathrm{~d} x+s \int_{\partial \Omega} v^{s-1} \frac{\partial v^{n}}{\partial v} \mathrm{~d} \xi+s \int_{\Omega} v^{s-1} \mathrm{~d} x \int_{\Omega} u^{q} \mathrm{~d} x \\
&=-\frac{4 m s(s-1)}{(s+m-1)^{2}} \int_{\Omega}\left|\nabla u^{\frac{s+m-1}{2}}\right|^{2} \mathrm{~d} x+s \int_{\partial \Omega} u^{s-1} \frac{\partial u^{m}}{\partial v} \mathrm{~d} \xi+s \int_{\Omega} u^{s-1} \mathrm{~d} x \int_{\Omega} v^{p} \mathrm{~d} x \\
& \quad-\frac{4 n s(s-1)}{(s+n-1)^{2}} \int_{\Omega}\left|\nabla u^{\frac{s+n-1}{2}}\right|^{2} \mathrm{~d} x+s \int_{\partial \Omega} v^{s-1} \frac{\partial v^{n}}{\partial v} \mathrm{~d} \xi+s \int_{\Omega} v^{s-1} \mathrm{~d} x \int_{\Omega} u^{q} \mathrm{~d} x .
\end{aligned}
$$

When the equations take Dirichlet boundary conditions of Eq. (1.4), Eq. (3.2) becomes

$$
J^{\prime}(t)=J_{1}^{\prime}(t)+J_{2}^{\prime}(t)
$$

where

$$
\begin{aligned}
& J_{1}^{\prime}(t)=\left[c_{1} \int_{\Omega}\left|\nabla u^{a}\right|^{2} \mathrm{~d} x+s \int_{\Omega} u^{s-1} \mathrm{~d} x \int_{\Omega} v^{p} \mathrm{~d} x\right], \\
& J_{2}^{\prime}(t)=\left[d_{1} \int_{\Omega}\left|\nabla v^{b}\right|^{2} \mathrm{~d} x+s \int_{\Omega} v^{s-1} \mathrm{~d} x \int_{\Omega} u^{q} \mathrm{~d} x\right], \\
& c_{1}=-\frac{4 m s(s-1)}{(s+m-1)^{2}}, \quad d_{1}=-\frac{4 n s(s-1)}{(s+n-1)^{2}}, \quad a=\frac{s+m-1}{2}, \quad b=\frac{s+n-1}{2} .
\end{aligned}
$$

First, Hölder inequality is used to estimate the second term of $J_{1}^{\prime}(t)$ in Eq. (3.3), and it is obtained that

$$
\begin{aligned}
& \int_{\Omega} u^{s-1} \mathrm{~d} x \leq\left(\int_{\Omega}\left(u^{s-1}\right)^{\frac{s}{s-p}} \mathrm{~d} x\right)^{\frac{s-p}{s}}|\Omega|^{\frac{p}{s}}, \\
& \int_{\Omega} v^{p} \mathrm{~d} x \leq\left(\int_{\Omega}\left(v^{p}\right)^{\frac{s}{p}} \mathrm{~d} x\right)^{\frac{p}{s}}|\Omega|^{\frac{s-p}{s}}, \\
& \int_{\Omega} u^{s-1} \mathrm{~d} x \int_{\Omega} v^{p} \mathrm{~d} x \leq\left(\int_{\Omega}\left(u^{s-1}\right)^{\frac{s}{s-p}} \mathrm{~d} x\right)^{\frac{s-p}{s}}\left(\int_{\Omega}\left(v^{p}\right)^{\frac{s}{p}} \mathrm{~d} x\right)^{\frac{p}{s}}|\Omega|,
\end{aligned}
$$


according to Young inequality and equation above, it is obtained that

$$
\int_{\Omega} u^{s-1} \mathrm{~d} x \int_{\Omega} v^{p} \mathrm{~d} x \leq \frac{|\Omega|(s-p)}{s} \int_{\Omega} u^{\frac{s(s-1)}{s-p}} \mathrm{~d} x+\frac{|\Omega| p}{s} \int_{\Omega} v^{s} \mathrm{~d} x .
$$

Second, Hölder inequality is used to estimate the first term on the right side of Eq. (3.4), and it is obtained that

$$
\int_{\Omega} u^{\frac{s(s-1)}{s-p}} \mathrm{~d} x=\int_{\Omega} u^{a} u^{\frac{s(s-1)}{s-p}-a} \mathrm{~d} x \leq\left(\int_{\Omega} u^{4 a} \mathrm{~d} x\right)^{\frac{1}{4}}\left(\int_{\Omega} u^{\frac{2\left[\frac{2 s(s-1)}{s-p}-(s+m-1)\right]}{3}} \mathrm{~d} x\right)^{\frac{3}{4}}
$$

from the second term on the right side of inequality sign of (3.5) and Hölder inequality, we can know

$$
\int_{\Omega} u^{\frac{2\left[\frac{2(s-1)}{s-p}-(s+m-1)\right]}{3}} \mathrm{~d} x \leq\left(\int_{\Omega} u^{s} \mathrm{~d} x\right)^{\frac{2\left[1+\frac{2(s-1)}{s-p}-\frac{m-1}{s}\right]}{3}} \mathrm{~d} x|\Omega|^{\frac{2\left[\frac{1}{2}-\frac{2(p-1)}{s-p}+\frac{m-1}{s}\right]}{3}},
$$

from the first term on the right side of inequality sign of (3.5) and Hölder inequality, we can know

$$
\int_{\Omega} u^{4 a} \mathrm{~d} x=\int_{\Omega} u^{a} u^{3 a} \mathrm{~d} x \leq\left(\int_{\Omega} u^{2 a} \mathrm{~d} x\right)^{\frac{1}{2}}\left(\int_{\Omega}\left(u^{a}\right)^{6} \mathrm{~d} x\right)^{\frac{1}{2}},
$$

using the following Sobolev inequality ([10]):

$$
\left(\int_{\Omega}|\phi|^{\gamma_{1}} \mathrm{~d} x\right)^{\frac{1}{\gamma_{1}}} \leq C\left(\int_{\Omega}|\nabla \phi|^{\gamma_{2}} \mathrm{~d} x\right)^{\frac{1}{\gamma_{2}}}
$$

where $\gamma_{1}=6, \gamma_{2}=2, C=4^{\frac{1}{3}} 3^{-\frac{1}{2}} \pi^{-\frac{2}{3}}$, the second term of Eq. (3.7) can be simplified to

$$
\left(\int_{\Omega}\left(u^{a}\right)^{6} \mathrm{~d} x\right)^{\frac{1}{2}} \leq C^{3}\left(\int_{\Omega}\left|\nabla u^{a}\right|^{2} \mathrm{~d} x\right)^{\frac{3}{2}},
$$

by synthesizing Eqs. (3.7) and (3.8), we have

$$
\left(\int_{\Omega} u^{4 a} \mathrm{~d} x\right)^{\frac{1}{4}} \leq C^{\frac{3}{4}}\left(\int_{\Omega} u^{2 a} \mathrm{~d} x\right)^{\frac{1}{8}}\left(\int_{\Omega}\left|\nabla u^{a}\right|^{2} \mathrm{~d} x\right)^{\frac{3}{8}}
$$

based on Lemma 2.1, Eq. (3.9) becomes

$$
\left(\int_{\Omega} u^{4 a} \mathrm{~d} x\right)^{\frac{1}{4}} \leq C^{\frac{3}{4}} \lambda^{-\frac{1}{8}}\left(\int_{\Omega}\left|\nabla u^{a}\right|^{2} \mathrm{~d} x\right)^{\frac{1}{2}} .
$$

Combining Eqs. (3.6) and (3.10), Eq. (3.5) becomes

$$
\int_{\Omega} u^{\frac{s(s-1)}{s-p}} \mathrm{~d} x \leq c_{2}\left(\int_{\Omega}\left|\nabla u^{a}\right|^{2} \mathrm{~d} x\right)^{\frac{1}{2}}\left(\int_{\Omega} u^{s} \mathrm{~d} x\right)^{w_{1}},
$$


where

$$
c_{2}=C^{\frac{3}{4}} \lambda^{-\frac{1}{8}}|\Omega|^{\frac{\frac{1}{2}-\frac{2(p-1)}{s-p}+\frac{m-1}{s}}{2}}, \quad w_{1}=\frac{1+\frac{2(p-1)}{s-p}-\frac{m-1}{s}}{2} .
$$

By synthesizing Eqs. (3.4)-(3.11), $J_{1}^{\prime}(t)$ of Eq. (3.3) becomes

$$
J_{1}^{\prime}(t) \leq c_{1} \int_{\Omega}\left|\nabla u^{a}\right|^{2} \mathrm{~d} x+c_{2}(s-p)\left(\theta_{1}\left[\int_{\Omega}\left|\nabla u^{a}\right|^{2} \mathrm{~d} x\right]^{\frac{1}{2}} \theta_{1}^{-1}\left[\left(\int_{\Omega} u^{s} \mathrm{~d} x\right)^{2 w_{1}}\right]^{\frac{1}{2}}\right)+p \int_{\Omega} v^{s} \mathrm{~d} x
$$

Using the fundamental inequality

$$
a^{q} b^{p} \leq q a+p b \quad(a, b>0, p, q \geq 0, p+q=1),
$$

Eq. (3.12) becomes

$$
J_{1}^{\prime}(t) \leq\left(c_{1}+\frac{c_{2}(s-p) \theta_{1}}{2}\right) \int_{\Omega}\left|\nabla u^{a}\right|^{2} \mathrm{~d} x+\frac{c_{2}(s-p) \theta_{1}^{-1}}{2}\left(\int_{\Omega} u^{s} \mathrm{~d} x\right)^{2 w_{1}}+p \int_{\Omega} v^{s} \mathrm{~d} x .
$$

The same derivation method is used to estimate the $J_{2}^{\prime}(t)$ term in Eq. (3.3):

$$
J_{2}^{\prime}(t) \leq\left(d_{1}+\frac{d_{2}(s-q) \theta_{2}}{2}\right) \int_{\Omega}\left|\nabla v^{b}\right|^{2} \mathrm{~d} x+\frac{d_{2}(s-q) \theta_{2}^{-1}}{2}\left(\int_{\Omega} v^{s} \mathrm{~d} x\right)^{2 w_{2}}+q \int_{\Omega} u^{s} \mathrm{~d} x,
$$

where

$$
d_{2}=C^{\frac{3}{4}} \lambda^{-\frac{1}{8}}|\Omega|^{\frac{1 \frac{2(q-1)}{2}+\frac{n-1}{s}}{2}}, \quad w_{2}=\frac{1+\frac{2(q-1)}{s-q}-\frac{n-1}{s}}{2} .
$$

In order to deal with the gradient terms in Eqs. (3.13) and (3.14), we set $\theta_{1}=-\frac{2 c_{1}}{c_{2}(s-p)}$ and $\theta_{2}=-\frac{2 d_{1}}{d_{2}(s-q)}$. Finally, by synthesizing Eqs. (3.13) and (3.14), we obtain

$$
J^{\prime}(t) \leq-\frac{c_{2}^{2}(s-p)^{2}}{4 c_{1}}\left(\int_{\Omega} u^{s} \mathrm{~d} x\right)^{2 w_{1}}-\frac{d_{2}^{2}(s-q)^{2}}{4 d_{1}}\left(\int_{\Omega} v^{s} \mathrm{~d} x\right)^{2 w_{2}}+p \int_{\Omega} v^{s} \mathrm{~d} x+q \int_{\Omega} u^{s} \mathrm{~d} x
$$

Take $K_{1}=-\frac{c_{2}^{2}(s-p)^{2}}{4 c_{1}}-\frac{d_{2}^{2}(s-q)^{2}}{4 d_{1}}, K_{2}=p+q, \beta_{1}=\max \left\{2 w_{1}, 2 w_{2}\right\}>1$. Eq. (3.14) becomes

$$
J^{\prime}(t) \leq K_{1} J^{\beta_{1}}(t)+K_{2} J(t)
$$

Integrating (3.16) from 0 to $t^{\star}$, we obtain

$$
t^{\star} \geq \int_{J(0)}^{\infty} \frac{\mathrm{d} \eta}{K_{1} \eta^{\beta_{1}}+K_{2} \eta} .
$$

This completes the proof of the theorem. 


\section{Lower bound of blow up time under Neumann boundary con- ditions}

The lower bound of blow up time for solutions of equations under the Neumann boundary conditions is discussed below.

\subsection{The case of $l>0$}

Theorem 4.1. Define the same measure as (3.1) and the same condition as s. If $(u, v)$ is a nonnegative classical solution to the equation set (1.1)-(1.2) with (1.3) and (1.5), then the lower bound of $t^{\star}$ is

$$
\int_{J(0)}^{\infty} \frac{\mathrm{d} \eta}{K_{1} \eta^{\beta_{1}}+K_{3} \eta^{\beta_{2}}+\left(K_{2}+K_{4}\right) \eta}
$$

where $J(0)=\int_{\Omega}\left(u_{0}^{s}+v_{0}^{s}\right) \mathrm{d} x$, the normal number $K_{1}, K_{2}, K_{3}, K_{4}, \beta_{1}, \beta_{2}$ is given in the following proof.

Proof. Lemma 2.2 is used to estimate two boundary terms in Eq. (3.2), then

$$
\int_{\partial \Omega} u^{s-1} \frac{\partial u^{m}}{\partial v} \mathrm{~d} \xi=l \int_{\Omega} u^{s} \mathrm{~d} \xi \leq \frac{3 l}{\rho_{0}} \int_{\Omega} u^{s} \mathrm{~d} x+\frac{s d}{\rho_{0}} \int_{\partial \Omega} u^{s-1}|\nabla u| \mathrm{d} x
$$

where $\rho_{0}=\min _{\partial \Omega}(\mathbf{x} \cdot \mathbf{n})>0, d=\max _{\partial \Omega}|\mathbf{x}|$.

From the second term on the right hand side of Eq. (4.1) and using Hölder inequality and Lemma 2.3, we can know

$$
\begin{aligned}
\int_{\Omega} u^{s-1}|\nabla u| \mathrm{d} x & \leq\left(\int_{\Omega} u^{s+m-3}|\nabla u|^{2} \mathrm{~d} x\right)^{\frac{1}{2}}\left(\int_{\Omega} u^{s-(m-1)} \mathrm{d} x\right)^{\frac{1}{2}} \\
& \leq \frac{r_{1}}{2}\left(\int_{\Omega} u^{s+m-3}|\nabla u|^{2} \mathrm{~d} x\right)+\frac{1}{2 r_{1}} \int_{\Omega} u^{s-(m-1)} \mathrm{d} x \\
& =-\frac{2 m s r_{1}(s-1)}{(s+m-1)^{2}} \int_{\Omega}\left|\nabla u^{\frac{s+m-1}{2}}\right|^{2} \mathrm{~d} x+\frac{1}{2 r_{1}} \int_{\Omega} u^{s-(m-1)} \mathrm{d} x
\end{aligned}
$$

where $r_{1}$ is an arbitrary constant.

The Hölder inequality is used to estimate the second term on the right hand side of Eq. (4.2). We then have

$$
\int_{\Omega} u^{s-(m-1)} \mathrm{d} x \leq\left(\int_{\Omega} u^{s} \mathrm{~d} x\right)^{\frac{s-(m-1)}{k}}|\Omega|^{\frac{m-1}{s}}
$$

Substituting (4.2) and (4.3) into Eq. (4.1), we get

$$
\int_{\partial \Omega} u^{s-1} \frac{\partial u^{m}}{\partial v} \mathrm{~d} \xi \leq \frac{3 l}{\rho_{0}} \int_{\Omega} u^{s} \mathrm{~d} x-c_{3} \int_{\Omega}\left|\nabla u^{a}\right|^{2} \mathrm{~d} x+c_{4}\left(\int_{\Omega} u^{s} \mathrm{~d} x\right)^{w_{3}}
$$


where $c_{3}=\frac{2 m s r_{1}(s-1)}{(s+m-1)^{2}}, c_{4}=\frac{s d}{2 r_{1} \rho_{0}}|\Omega|^{\frac{m-1}{s}}, w_{3}=\frac{s-(m-1)}{s}$.

Similarly, another boundary term in Eq. (3.2) is estimated as follows

$$
\int_{\partial \Omega} v^{s-1} \frac{\partial u^{m}}{\partial v} \mathrm{~d} \xi \leq \frac{3 l}{\rho_{0}} \int_{\Omega} v^{s} \mathrm{~d} x-d_{3} \int_{\Omega}\left|\nabla u^{b}\right|^{2} \mathrm{~d} x+d_{4}\left(\int_{\Omega} v^{s} \mathrm{~d} x\right)^{w_{4}}
$$

where $d_{3}=-\frac{2 n s r_{2}(s-1)}{(s+n-1)^{2}}, d_{4}=\frac{s d}{2 r_{2} \rho_{0}}|\Omega|^{\frac{n-1}{s}}, w_{4}=\frac{s-(n-1)}{s}, r_{2}$ is an arbitrary constant.

Substituting (3.16), (4.4) and (4.5) into Eq. (3.2), we get

$$
\begin{aligned}
J^{\prime}(t) \leq( & \left.c_{1}-c_{3}+\frac{c_{2}(s-p) \theta_{3}}{2}\right) \int_{\Omega}\left|\nabla u^{a}\right|^{2} \mathrm{~d} x+\left(d_{1}-d_{3}+\frac{d_{2}(s-q) \theta_{4}}{2}\right) \int_{\Omega}\left|\nabla v^{b}\right|^{2} \mathrm{~d} x \\
& +c_{4}\left(\int_{\Omega} u^{s} \mathrm{~d} x\right)^{w_{3}}+c_{5}\left(\int_{\Omega} u^{s} \mathrm{~d} x\right)^{2 w_{1}}+\left(\frac{3 l}{\rho_{0}}+q\right) \int_{\Omega} u^{s} \mathrm{~d} x \\
& +d_{4}\left(\int_{\Omega} v^{s} \mathrm{~d} x\right)^{w_{4}}+d_{5}\left(\int_{\Omega} v^{s} \mathrm{~d} x\right)^{2 w_{2}}+\left(\frac{3 l}{\rho_{0}}+p\right) \int_{\Omega} v^{s} \mathrm{~d} x
\end{aligned}
$$

where

$$
\begin{aligned}
& c_{5}=-\frac{c_{2}^{2}(s-p)^{2}}{4 c_{1}}, d_{5}=-\frac{d_{2}^{2}(s-q)^{2}}{4 d_{1}}, \\
& c_{1}-c_{3}+\frac{c_{2}(s-p) \theta_{3}}{2}=0, d_{1}-d_{3}+\frac{d_{2}(s-q) \theta_{4}}{2}=0, \\
& K_{1}=c_{5}+d_{5}, K_{2}=p+q, K_{3}=c_{4}+d_{4}, K_{4}=\frac{3 l}{\rho_{0}}, \\
& \beta_{1}=\max \left\{2 w_{1}, 2 w_{2}\right\}>1, \quad \beta_{2}=\max \left\{w_{1}, w_{2}\right\}>0 .
\end{aligned}
$$

The Eq. (4.6) becomes

$$
J^{\prime}(t) \leq K_{1} J^{\beta_{1}}(t)+K_{3} J^{\beta_{2}}(t)+\left(K_{2}+K_{4}\right) J(t) .
$$

Integrating (4.7) from 0 to $t^{\star}$, we obtain

$$
t^{\star} \geq \int_{J(0)}^{\infty} \frac{\mathrm{d} \eta}{K_{1} \eta^{\beta_{1}}+K_{3} \eta^{\beta_{2}}+\left(K_{2}+K_{4}\right) \eta}
$$

where $J(0)=\int_{\Omega}\left(u^{0}+v^{0}\right) \mathrm{d} x$.

\subsection{The case of $l \leq 0$}

If $l \leq 0$, then $\frac{\partial u^{m}}{\partial v} \leq 0, \frac{\partial v^{n}}{\partial v} \leq 0$, according to Eq. (3.2), we obtain

$$
J^{\prime}(t) \leq J_{1}^{\prime}(t)+J_{2}^{\prime}(t)
$$

That is, the same measure relation is obtained with Eq. (3.3). Therefore, when $l \leq 0$, the lower bound of blow up time of the equation set (1.1)-(1.2) with (1.3) and (1.5) is consistent with that of Eq. (3.17). 


\section{Acknowledgments}

This work is supported by Natural Science Basic Research Project of Shaanxi Province (2019JM-534); Soft Science Project of Shaanxi Province (2019KRM169); Planned Projects of the 13th Five-year Plan for Education Science of Shaanxi Province (SGH18H544); Project on Higher Education Teaching Reform of Xi'an International University (2019B36), and the Youth Innovation Team of Shaanxi Universities. The author would like to deeply thank all the reviewers for their insightful and constructive comments.

\section{References}

[1] Liu D. M., Mu C. L., Xin Q., Lower bounds estimate for the blow-up time of a nonlinear nonlocal porous medium equation. Acta Mathematica Scientia, 32B (3) (2012) 1206-1212.

[2] Liu Y., Blow-up phenomena for the nonlinear nonlocal porous medium equation under Robin boundary condition. Computers and Mathematics with Applications, 66 (2013) 2092-2095.

[3] Fang Z. B., Chai Y., Lower bounds estimates of the blow-up time for a nonlinear nonlocal porous medium equation with Neumann boundary condition. Periodical of Ocean University of China, 46(9) (2016) 129-132.

[4] Payne L. E., Philippin G. A., Blow-up phenomena for a Class of Parabolic systems with Time Dependent Coefficients. Applied Mathematics, 3 (2012) 325-330.

[5] Tao X. Y., Fang Z. B., Blow-up phenomena for a nonlinear reactionCdiffusion system with time dependent coefficients. Computers and Mathematics with Applications, 74 (2017) 25202528.

[6] Ma L. W., Fang Z. B., Blow-up phenomena for a semilinear parabolic equation with weighted inner absorption under nonlinear boundary flux. Math. Methods Appl. Sci., 40 (2017) 115-128.

[7] Bao A. G., Song X. F., Bounds for the blowup time of the solution to a parabolic system with nonlocal factors in nonlinearities. Comput. Math. Appl., 71(3) (2016) 723-729.

[8] Wang N., Song X. F., Lv X. S., Estimates for the blowup time of a combustion model with nonlocal heat sources. J. Math. Anal. Appl., 436 (2) (2016) 1180-1195.

[9] Li Y. F., Liu Y., Lin C. H., Blow-up phenomena for a nonlinear reactionCdiffusion system with time dependent coefficients. Nonlinear Analysis, 11 (2010) 3815-3823.

[10] Talenti G., Best constant in Sobolev inequality. Ann. Mat. Pura Appl., 110 (1976) 353-372. 